Georgian Mathematical Journal

1(1994), No. 6, 587-598

\title{
NON-STATIONARY PROBLEMS OF GENERALIZED ELASTOTHERMODIFFUSION FOR INHOMOGENEOUS MEDIA
}

\author{
T. BURCHULADZE
}

\begin{abstract}
The method of investigation of non-stationary boundary value problems of the theory of thermodiffusion using the Laplace integral transform is described. In the classical theory of elasticity this method was first used by V. Kupradze and the author.
\end{abstract}

The interconnection of deformation, thermal conductivity and diffusion processes in an elastic isotropic solid body is described by a system of five scalar partial differential equations of general type. In the classical case this system is hyperbolic with respect to some part of components of an unknown vector function and parabolic with respect to the rest components. A system of equations of the conjugate (connected) theory of thermoelasticity is a particular case [1-4].

In the classical theory of elastothermodiffusion it is assumed that propagation velocity of heat and of diffusing substance is infinitely large.

In particular, however, it is often necessary to take into account the fact that heat propagates not with an infinitely large but with a finite velocity. The heat flux does not occur in the body instantly but is characterized by the finite relaxation time.

The consideration of these physical factors makes the main system of differential equations very complicated. There exist various generalizations of this theory. Three-dimensional non-stationary problems of non-classical (generalized) thermodiffusion are treated in [5-8].

In this paper the Green-Lindsay theory is generalized to problems of elastothermodiffusion. Initial boundary value problems are investigated for the considered physical system of differential equations in piecewisehomogeneous media with boundary and contact conditions; a substantiation of the Riesz-Fischer-Kupradze method is given and approximate solutions are considered.

1991 Mathematics Subject Classification. 73B30, 73C25. 
Let us consider a three-dimensional homogeneous isotropic elastic medium in which a thermodiffusion process takes place. The deformed state is described by the displacement vector $v(x, t)=\left(v_{1}, v_{2}, v_{3}\right)=\left\|v_{k}\right\|_{3 \times 1}$ (onecolumn matrix), the temperature change $v_{4}(x, t)$ and the "chemical potential" of the medium $v_{5}(x, t) ; C(x, t)=\gamma_{2} \operatorname{div} v(x, t)+a_{12} v_{4}(x, t)+a_{2} v_{5}(x, t)$, where $C(x, t)$ is the diffusing substance concentration; $x=\left(x_{1}, x_{2}, x_{3}\right)$ is a point in the Euclidean space $\mathbb{R}^{3}, t \geq 0$ is the time and $X=\left(X_{1}, X_{2}, X_{3}\right)$, $X_{4}, X_{5}$ are the given functions. We consider a system of partial differential equations of the generalized elastothermodiffusion theory written in the form

$$
\begin{aligned}
A\left(\frac{\partial}{\partial x}\right) v- & \sum_{k=1}^{2} \gamma_{k} \operatorname{grad} v_{3+k}+X=\rho \frac{\partial^{2} v}{\partial t^{2}}+ \\
+ & \tau^{1} \sum_{k=1}^{2} \gamma_{k} \frac{\partial}{\partial t} \operatorname{grad} v_{3+k}, \\
\delta_{1} \Delta v_{4}+X_{4}= & a_{1}\left(1+\tau^{0} \frac{\partial}{\partial t}\right) \frac{\partial v_{4}}{\partial t}+\gamma_{1} \frac{\partial}{\partial t} \operatorname{div} v+ \\
& +a_{12}\left(1+\tau^{0} \frac{\partial}{\partial t}\right) \frac{\partial v_{5}}{\partial t}, \\
\delta_{2} \Delta v_{5}+X_{5}= & a_{2}\left(1+\tau^{0} \frac{\partial}{\partial t}\right) \frac{\partial v_{5}}{\partial t}+\gamma_{2} \frac{\partial}{\partial t} \operatorname{div} v+ \\
& +a_{12}\left(1+\tau^{0} \frac{\partial}{\partial t}\right) \frac{\partial v_{4}}{\partial t},
\end{aligned}
$$

where $A\left(\frac{\partial}{\partial x}\right) \equiv\left\|\mu \delta_{j k} \Delta+(\lambda+\mu) \frac{\partial^{2}}{\partial x_{j} \partial x_{k}}\right\|_{3 \times 3}$ is the statical operator of Lamé [8], $\delta_{j k}$ being the Kroneker symbol. The elastic, thermal, diffusion and relaxation constants satisfy the natural restrictions

$$
\begin{gathered}
\mu>0, \quad 3 \lambda+2 \mu>0, \quad \rho>0, \quad a_{k}>0, \quad \delta_{k}>0, \quad \gamma_{k}>0, \quad k=1,2 \\
a_{1} a_{2}-a_{12}^{2}>0, \quad \tau^{1} \geq \tau^{0}>0 .
\end{gathered}
$$

In particular, for relaxation constants $\tau^{1}=\tau^{0}=0$ we have the classical case.

Let $D_{1} \subset \mathbb{R}^{3}$ be a finite domain bounded by the closed Liapunov surface $S$ and $D_{2}=\mathbb{R}^{3} \backslash \bar{D}_{1}$ be an infinite domain, $n=\left(n_{1}, n_{2}, n_{3}\right)$ is the unit normal on $S$. Elastothermodiffusion constants of the domain $D_{j}$ will be denoted by the left-hand subscripts ${ }_{j} \lambda,{ }_{j} \mu,{ }_{j} \rho,{ }_{j} \tau^{0},{ }_{j} \tau^{1}, \ldots, j=1,2$.

Problem $A^{t}$. Define in the infinite cylinder $Z_{\infty}=\left\{(x, t): x \in D_{1} \cup\right.$ $\left.D_{2}, t \in\right] 0, \infty[\}$ the regular vetor $V=\left(v, v_{4}, v_{5}\right) \in C^{1}\left(\bar{Z}_{\infty}\right) \cap C^{2}\left(Z_{\infty}\right)$ from the conditions

$$
\forall(x, t) \in Z_{\infty}:{ }_{j} \mu \Delta v(x, t)+\left({ }_{j} \lambda+{ }_{j} \mu\right) \operatorname{grad} \operatorname{div} v-
$$




$$
\begin{gathered}
-\sum_{k=1}^{2}{ }_{j} \gamma_{k} \operatorname{grad} v_{3+k}+{ }_{j} X_{j}=\rho \frac{\partial^{2} v}{\partial t^{2}}+{ }_{j} \tau^{1} \sum_{k=1}^{2}{ }_{j} \gamma_{k} \frac{\partial}{\partial t} \operatorname{grad} v_{3+k} \\
{ }_{j} \delta_{1} \Delta v_{4}(x, t)+{ }_{j} X_{4}={ }_{j} a_{1}\left(1+{ }_{j} \tau^{0} \frac{\partial}{\partial t}\right) \frac{\partial v_{4}}{\partial t}+ \\
+{ }_{j} \gamma_{1} \frac{\partial}{\partial t} \operatorname{div} v+{ }_{j} a_{12}\left(1+{ }_{j} \tau^{0} \frac{\partial}{\partial t}\right) \frac{\partial v_{5}}{\partial t} \\
{ }_{j} \delta_{2} \Delta v_{5}(x, t)+{ }_{j} X_{5}={ }_{j} a_{2}\left(1+{ }_{j} \tau^{0} \frac{\partial}{\partial t}\right) \frac{\partial v_{5}}{\partial t}+ \\
+{ }_{j} \gamma_{2} \frac{\partial}{\partial t} \operatorname{div} v+{ }_{j} a_{12}\left(1+{ }_{j} \tau^{0} \frac{\partial}{\partial t}\right) \frac{\partial v_{4}}{\partial t} \\
\forall x \in D_{j}:{ }_{t \rightarrow+0} \lim _{t} V(x, t)={ }_{j} \varphi^{(0)}(x), \quad j=1,2, \\
\lim _{t \rightarrow+0} \frac{\partial V(x, t)}{\partial t}={ }_{j} \varphi^{(1)}(x), \quad j=1,2 \\
\forall(y, t) \in S^{\infty} \equiv\{(y, t): y \in S, t \in[0, \infty[\}: \\
{[V]_{S}^{ \pm} \equiv V^{+}(y, t)-V^{-}(y, t)=f(y, t)} \\
{[R V]_{S}^{ \pm} \equiv\left[{ }_{1} R\left(\frac{\partial}{\partial y}, n\right) V(y, t)\right]^{+}-} \\
-\left[{ }_{2} R\left(\frac{\partial}{\partial y}, n\right) V(y, t)\right]^{-}=F(y, t)
\end{gathered}
$$

for large values of $t$ and $x \in D_{2}$ :

$$
\begin{gathered}
\left|D_{x, t}^{\alpha} V(x, t)\right| \leq \frac{\text { const }}{1+|x|^{1+|\alpha|}} e^{\sigma_{0} t}, \quad|\alpha|=\overline{0,2}, \quad \sigma_{0} \geq 0, \\
D_{x, t}^{\alpha} \equiv \frac{\partial^{|\alpha|}}{\partial x_{1}^{\alpha_{1}} \partial x_{2}^{\alpha_{2}} \partial x_{3}^{\alpha_{3}} \partial t^{\alpha_{4}}}, \quad|\alpha|=\sum_{k=1}^{4} \alpha_{k},
\end{gathered}
$$

where $\alpha=\left(\alpha_{1}, \alpha_{2}, \alpha_{3}, \alpha_{4}\right)$ is a multi-index;

$$
\begin{aligned}
& { }_{j} \varphi^{(0)}(x)=\left({ }_{j} \varphi_{1}^{(0)},{ }_{j} \varphi_{2}^{(0)},{ }_{j} \varphi_{3}^{(0)},{ }_{j} \varphi_{4}^{(0)},{ }_{j} \varphi_{5}^{(0)}\right), \\
& { }_{j} \varphi^{(1)}(x)=\left({ }_{j} \varphi_{1}^{(1)},{ }_{j} \varphi_{2}^{(1)},{ }_{j} \varphi_{3}^{(1)},{ }_{j} \varphi_{4}^{(1)},{ }_{j} \varphi_{5}^{(1)}\right), \\
& f(y, t)=\left(f_{1}, f_{2}, f_{3}, f_{4}, f_{5}\right), \\
& F(y, t)=\left(F_{1}, F_{2}, F_{3}, F_{4}, F_{5}\right), \quad t \geq 0, \quad y \in S,
\end{aligned}
$$

are the given real functions; ${ }_{j} R\left(\frac{\partial}{\partial x}, n\right)$ is a stress operator in the thermodiffusion theory for the medium $D_{j}(5 \times 5$ matrix $)$ :

$$
{ }_{j} R\left(\frac{\partial}{\partial x}, n\right)=\left\|_{j} R_{k l}\right\|_{k, l=\overline{1,5}},
$$


where

$$
\begin{gathered}
{ }_{j} R_{k l}={ }_{j} \mu \delta_{l k} \frac{\partial}{\partial n(x)}+{ }_{j} \lambda n_{l}(x) \frac{\partial}{\partial x_{k}}+{ }_{j} \mu n_{k}(x) \frac{\partial}{\partial x_{l}}, \quad k, l=\overline{1,3}, \\
{ }_{j} R_{k l}=-{ }_{j} \gamma_{l-3} n_{k}\left(1+{ }_{j} \tau^{l-3} \frac{\partial}{\partial t}\right), \quad k=\overline{1,3}, \quad l=4,5, \\
{ }_{j} R_{k l}={ }_{j} \delta_{k-3} \delta_{k l} \frac{\partial}{\partial n(x)}, \quad k=4,5, \quad l=\overline{1,5},
\end{gathered}
$$

here $n(x)$ is $C^{\infty}$-extention of $n$ onto $\mathbb{R}^{3}$;

$$
\begin{aligned}
& V^{+}(y, t)=\lim _{D_{1} \ni x \rightarrow y \in S} V(x, t), \quad V^{-}(y, t)=\lim _{D_{2} \ni x \rightarrow y \in S} V(y, t), \\
& {\left[{ }_{1} R\left(\frac{\partial}{\partial y}, n(y)\right) V(y, t)\right]^{+}=\lim _{D_{1} \ni x \rightarrow y \in S} R\left(\frac{\partial}{\partial x}, n(y)\right) V(x, t),} \\
& {\left[{ }_{2} R\left(\frac{\partial}{\partial y}, n(y)\right) V(y, t)\right]^{-}=\lim _{D_{2} \ni x \rightarrow y \in S} R\left(\frac{\partial}{\partial x}, n(y)\right) V(x, t) .}
\end{aligned}
$$

It is easy to verify that

$$
\begin{aligned}
R\left(\frac{\partial}{\partial x}, n\right) V= & \left(T v-\gamma_{1}\left(1+\tau^{1} \frac{\partial}{\partial t}\right) n v_{4}-\right. \\
& \left.\gamma_{2}\left(1+\tau^{1} \frac{\partial}{\partial t}\right) n v_{5}, \delta_{1} \frac{\partial v_{4}}{\partial n}, \delta_{2} \frac{\partial v_{5}}{\partial n}\right)
\end{aligned}
$$

where $T$ is the "classical" stress operator.

For a classical (regular) solution to exist, it is necessary that the conditions of "natural compatibility" of initial data be fulfilled. These conditions have the form

$$
\begin{aligned}
\forall y \in S: & { }_{1} \varphi^{(0)}(y)-{ }_{2} \varphi^{(0)}(y)=f(y, 0) \\
& { }_{1} \varphi^{(1)}(y)-{ }_{2} \varphi^{(1)}(y)=\lim _{t \rightarrow+0} \frac{\partial f(y, t)}{\partial t}, \\
{ }_{1} R\left(\frac{\partial}{\partial y}, n\right){ }_{1} \varphi^{(0)}(y)-{ }_{2} R\left(\frac{\partial}{\partial y}, n\right){ }_{2} \varphi^{(1)}(y) & =F(y, 0), \\
{ }_{1} R\left(\frac{\partial}{\partial y}, n\right){ }_{1} \varphi^{(1)}(y)-{ }_{2} R\left(\frac{\partial}{\partial y}, n\right){ }_{2} \varphi^{(1)}(y) & =\lim _{t \rightarrow+0} \frac{\partial F(y, t)}{\partial t} .
\end{aligned}
$$

The dynamic Problem $A^{t}$ is investigated by the Laplace transform method. However, the "natural compatibility" conditions of this method are not sufficient for our purpose and should therefore be complemented with 
"higher order compatibility" conditions. The latter have the form

$$
\begin{aligned}
& \left.\frac{\partial^{m} f(y, t)}{\partial t^{m}}\right|_{t=0}={ }_{1} \varphi^{(m)}(y)-{ }_{2} \varphi^{(m)}(y), \\
& \left.\frac{\partial^{m} F(y, t)}{\partial t^{m}}\right|_{t=0}={ }_{1} R_{1} \varphi^{(m)}(y)-{ }_{2} R{ }_{2} \varphi^{(m)}(y),
\end{aligned}
$$

where

$$
\begin{gathered}
{ }_{j} \varphi^{(m)}(x) \equiv\left({ }_{j} \varphi_{1}^{(m)}(x),{ }_{j} \varphi_{2}^{(m)}(x),{ }_{j} \varphi_{3}^{(m)}(x)\right)= \\
={ }_{j} \rho^{-1}\left[{ }_{j} \mu \Delta\left({ }_{j} \varphi_{1}^{(m-2)},{ }_{j} \varphi_{2}^{(m-2)},{ }_{j} \varphi_{3}^{(m-2)}\right)+\right. \\
+\left({ }_{j} \lambda+{ }_{j} \mu\right) \operatorname{grad} \operatorname{div}\left({ }_{j} \varphi_{1}^{(m-2)},{ }_{j} \varphi_{2}^{(m-2)},{ }_{j} \varphi_{3}^{(m-2)}\right)- \\
-{ }_{j} \gamma_{1} \operatorname{grad}{ }_{j} \varphi_{4}^{(m-2)}-{ }_{j} \gamma_{1 j} \tau^{1} \operatorname{grad}{ }_{j} \varphi_{4}^{(m-1)}- \\
\left.-{ }_{j} \gamma_{2} \operatorname{grad}{ }_{j} \varphi_{5}^{(m-2)}-{ }_{j} \gamma_{2 j} \tau^{1} \operatorname{grad}{ }_{j} \varphi_{5}^{(m-1)}+\left.\frac{\partial^{m-2}{ }_{j} X}{\partial t^{m-2}}\right|_{t=0}\right], \\
{ }_{j} a_{1 j} \tau^{0}{ }_{j} \varphi_{4}^{(m)}(x)+{ }_{j} a_{12 j} \tau^{0}{ }_{j} \varphi_{5}^{(m)}(x)={ }_{j} \delta_{1} \Delta_{j} \varphi_{4}^{(m-2)}-{ }_{j} a_{1 j} \varphi_{4}^{(m-1)}- \\
-{ }_{j} a_{12 j} \varphi_{5}^{(m-1)}-{ }_{j} \gamma_{1} \operatorname{div}\left({ }_{j} \varphi_{1}^{(m-1)},{ }_{j} \varphi_{2}^{(m-1)},{ }_{j} \varphi_{3}^{(m-1)}\right)+\left.\frac{\partial^{m-2}{ }_{j} X_{4}}{\partial t^{m-2}}\right|_{t=0}, \\
{ }_{j} a_{12 j} \tau^{0}{ }_{j} \varphi_{4}^{(m)}(x)+{ }_{j} a_{2 j} \tau^{0}{ }_{j} \varphi_{5}^{(m)}(x)={ }_{j} \delta_{2} \Delta_{j} \varphi_{5}^{(m-2)}-{ }_{j} a_{2 j} \varphi_{5}^{(m-1)}- \\
-{ }_{j} a_{12 j} \varphi_{4}^{(m-1)}-{ }_{j} \gamma_{2} \operatorname{div}\left({ }_{j} \varphi_{1}^{(m-1)},{ }_{j} \varphi_{2}^{(m-1)},{ }_{j} \varphi_{3}^{(m-1)}\right)+\left.\frac{\partial^{m-2}{ }_{j} X_{5}}{\partial t^{m-2}}\right|_{t=0} .
\end{gathered}
$$

These conditions of "quantitative nature" are sufficient for the existence of the classical solution. We will not dwell on this here but proceed to the construction of approximate solutions by the Riesz-Fischer-Kupradze method.

Theorem. If the initial data of Problem $A^{t}$ satisfy the above-given "higher order compatibility" conditions, then Problem $A^{t}$ has the unique classical solution which is represented by the Laplace-Mellin integral

$$
V(x, t)=\frac{1}{2 \pi i} \int_{\sigma-i \infty}^{\sigma+i \infty} e^{\zeta t} \widehat{V}(x, \zeta) d \zeta
$$

where $\widehat{V}(x, \zeta)$ is the solution of the corresponding problem for elliptic system represented in the form

$$
\widehat{V}(x, \zeta)=\sum_{k=0}^{\infty} a_{k}(\zeta) \stackrel{k}{\Omega}(x, \zeta)+\Omega(x, \zeta)
$$


The series converges uniformly; $a_{k}(\zeta), \stackrel{k}{\Omega}(x, \zeta), \Omega(x, \zeta)$ are the given vectorfunctions (constructed explicitly) and $\zeta=\sigma+i q$, where $\sigma \geq \sigma_{0}^{*}>\sigma_{0} ; \sigma_{0}^{*}$ is the defined constant.

Consider the Laplace transform

$$
\widehat{V}(x, \zeta)=\int_{0}^{\infty} e^{-\zeta t} V(x, t) d t
$$

where $\zeta=\sigma+i q$ is a complex parameter.

Using formally transform (4), the dynamic problem $A^{t}$ is reduced to the corresponding problem with the complex parameter $\zeta$ (spectral problem) for $\widehat{V}(x, \zeta)$.

Problem $A(\zeta)$. Define for each $\zeta \in \Pi_{\sigma_{0}^{*}} \equiv\left\{\zeta: \operatorname{Re} \zeta>\sigma_{0}^{*}>\sigma_{0}\right\}$ in $D=D_{1} \cup D_{2}$ the regular vector $\left.\widehat{V}=\left(\widehat{v}, \widehat{v}_{4}, \widehat{V}_{5}\right)\right)=\widehat{V}(\cdot, \zeta) \in C^{1}\left(\overline{D_{1} \cup D_{2}}\right) \cap$ $C^{2}\left(D_{1} \cup D_{2}\right)$ from the conditions

$$
\begin{aligned}
& \forall x \in D_{j}, \quad j=1,2: \\
& { }_{j} \mu \Delta \widehat{v}+\left({ }_{j} \lambda+{ }_{j} \mu\right) \operatorname{grad} \operatorname{div} v- \\
& -\sum_{k=1}^{2}{ }_{j} \gamma_{k}\left(1+{ }_{j} \tau^{1} \zeta\right) \operatorname{grad} \widehat{v}_{3+k}-{ }_{j} \rho \zeta^{2} \widehat{v}={ }_{j} \tilde{X}, \\
& { }_{j} \delta_{1} \Delta \widehat{v}_{4}-{ }_{j} a_{1}\left(1+{ }_{j} \tau^{0} \zeta\right) \widehat{v}_{4}-{ }_{j} a_{12} \zeta\left(1+{ }_{j} \tau^{0} \zeta\right) \widehat{v}_{5}- \\
& -{ }_{j} \gamma_{1} \zeta \operatorname{div} \widehat{v}={ }_{j} \widetilde{X}_{4}, \\
& { }_{j} \delta_{2} \Delta \widehat{v}_{5}-{ }_{j} a_{2}\left(1+{ }_{j} \tau^{0} \zeta\right) \widehat{v}_{5}-{ }_{j} a_{12} \zeta\left(1+{ }_{j} \tau^{0} \zeta\right) \widehat{v}_{4}- \\
& -{ }_{j} \gamma_{2} \zeta \operatorname{div} \widehat{v}={ }_{j} \widetilde{X}_{5}, \\
& \left|D_{x}^{\beta} \widehat{V}(x, \zeta)\right| \leq \frac{\text { const }}{1+|x|^{1+|\beta|}}, \quad|\beta|=\overline{0,2},
\end{aligned}
$$

where $\beta=\left(\beta_{1}, \beta_{2} \beta_{3}\right)$ is a multi-index,

$$
\begin{aligned}
{ }_{j} \widetilde{X} & =-{ }_{j} \widehat{X}-{ }_{j} \rho\left({ }_{j} \varphi_{1}^{(1)},{ }_{j} \varphi_{2}^{(1)},{ }_{j} \varphi_{3}^{(1)}\right)- \\
& -{ }_{j} \rho \zeta\left({ }_{j} \varphi_{1}^{(0)},{ }_{j} \varphi_{2}^{(0)},{ }_{j} \varphi_{3}^{(0)}\right)-\sum_{k=1}{ }_{j} \gamma_{k j} \operatorname{grad}{ }_{j} \varphi_{3+k}^{(0)}, \\
\tilde{X}_{4} & =-{ }_{j} \widehat{X}_{4}-{ }_{j} a_{1 j} \varphi_{4}^{(0)}-{ }_{j} a_{1 j} \tau^{0}\left({ }_{j} \varphi_{4}^{(1)}+\zeta_{j} \varphi_{4}^{(0)}\right)- \\
& -{ }_{j} a_{12}{ }_{j} \varphi_{5}^{(0)}-{ }_{j} a_{12} \tau^{0}{ }^{0}\left({ }_{j} \varphi_{5}^{(1)}+\zeta_{j} \varphi_{5}^{(0)}\right)- \\
& -\gamma_{1} \operatorname{div}\left({ }_{j} \varphi_{1}^{(0)},{ }_{j} \varphi_{2}^{(0)},{ }_{j} \varphi_{3}^{(0)}\right) \\
{ }_{j} \widetilde{X}_{5} & =-{ }_{j} \widehat{X}_{5}-{ }_{j} a_{2 j} \varphi_{5}^{(0)}-{ }_{j} a_{2 j} \tau^{0}\left({ }_{j} \varphi_{5}^{(1)}+\zeta_{j} \varphi_{5}^{(0)}\right)-
\end{aligned}
$$




$$
\begin{aligned}
& -{ }_{j} a_{12 j} \varphi_{4}^{(0)}-{ }_{j} a_{12 j} \tau^{0}\left({ }_{j} \varphi_{4}^{(1)}+\zeta_{j} \varphi_{4}^{(0)}\right)- \\
& -{ }_{j} \gamma_{2} \operatorname{div}\left({ }_{j} \varphi_{1}^{(0)},{ }_{j} \varphi_{2}^{(0)},{ }_{j} \varphi_{3}^{(0)}\right) \\
\forall y \in S: & \widehat{V}^{+}(y, \zeta)-\widehat{V}^{-}(y, \zeta)=\widehat{f}(y, \zeta) \\
& {\left[{ }_{1} R\left(\frac{\partial}{\partial y}, n\right) \widehat{V}(y, \zeta)\right]^{+}-\left[{ }_{2} R\left(\frac{\partial}{\partial y}, n\right) \widehat{V}(y, \zeta)\right]^{-}=\widetilde{F}(y, \zeta), } \\
\widetilde{F}(y, \zeta)= & \widehat{F}(y, \zeta)-{ }_{1} \gamma_{1} \tau^{1} n(y){ }_{1} \varphi_{4}^{(0)}+{ }_{2} \gamma_{1}{ }_{2} \tau^{1} n(y){ }_{2} \varphi_{4}^{(0)}- \\
& -{ }_{1} \gamma_{2}{ }_{1} \tau^{1} n(y){ }_{1} \varphi_{5}^{(0)}+{ }_{2} \gamma_{2}{ }_{2} \tau^{1} n(y){ }_{2} \varphi_{5}^{(0)}, \\
{ }_{j} \widehat{R}\left(\frac{\partial}{\partial y}, n\right) \widehat{V} & =\left(T \widehat{v}-n(y) \sum_{k=1}^{2}{ }_{j} \gamma_{k}\left(1+{ }_{j} \tau^{1} \zeta\right) \widehat{v}_{3+k},{ }_{j} \delta_{1} \frac{\partial \widehat{v}_{4}}{\partial n},{ }_{j} \delta_{2} \frac{\partial \widehat{v}_{5}}{\partial n}\right) .
\end{aligned}
$$

Let $L\left(\frac{\partial}{\partial x}, \zeta\right)$ be a matrix differential operator of Problem $A(\zeta)$ and $\Phi(x, \zeta)$ $=\left\|\Phi_{j k}(x, \zeta)\right\|_{5 \times 5}=\|\stackrel{1}{\Phi}, \stackrel{2}{\Phi}, \ldots, \stackrel{5}{\Phi}\|_{5 \times 5}$ be a matrix of fundamental solutions of this operator $L\left(\frac{\partial}{\partial x}, \zeta\right), \stackrel{k}{\Phi}(x, \zeta)=\left(\Phi_{1 k}, \Phi_{2 k}, \ldots, \Phi_{5 k}\right), k=\overline{1,5}$, be column vectors. The matrix $\Phi(x, \zeta)$ is constructed explicitly in terms of elementary functions [8]. Namely:

$$
\begin{gathered}
\Phi(x, \zeta) \equiv \widehat{L}\left(\frac{\partial}{\partial x}, \zeta\right) \varphi(x, \zeta) \equiv \\
\equiv \widehat{L}_{0}\left(\frac{\partial}{\partial x}, \zeta\right)\left(\Delta+\lambda_{4}^{2}\right) \varphi(x, \zeta) \equiv \widehat{L}_{0}\left(\frac{\partial}{\partial x}, \zeta\right) \widehat{\varphi}(x, \zeta) \\
\widehat{\varphi}(x, \zeta)=\sum_{k=1}^{4} c_{k} \frac{\exp \left(i \lambda_{k}|x|\right)}{|x|}
\end{gathered}
$$

where $\lambda_{k}, c_{k}, k=\overline{1,4}$ are constants, $\widehat{L}\left(\frac{\partial}{\partial x}, \zeta\right)$ is a matrix connected with $L\left(\frac{\partial}{\partial x}, \zeta\right): \widehat{L} L \equiv L \widehat{L} \equiv I \cdot \operatorname{det} L, I$ is the unit $5 \times 5$ matrix.

In the above assumptions the sense of the notations ${ }_{j} L\left(\frac{\partial}{\partial x}, \zeta\right)$ and ${ }_{j} \Phi(x, \zeta)$ becomes quite clear.

Thus we have to construct the solution of

Problem $A(\zeta)$.

$$
\begin{gathered}
\widehat{V}=\left(\widehat{v}, \widehat{v}_{4}, \widehat{v}_{5}\right) \in C^{1}(\bar{D}) \cap C^{2}(D), \\
\forall x \in D_{j}:{ }_{j} L\left(\frac{\partial}{\partial x}, \zeta\right) \widehat{V}(x, \zeta)={ }_{j} \chi(x), \quad j=1,2, \\
\forall y \in S:[\widehat{V}]_{S}^{ \pm} \equiv \widehat{V}^{+}(y, \zeta)-\widehat{V}^{-}(y, \zeta)=\widehat{f}(y, \zeta), \\
{[\widehat{R} \widehat{V}]_{S}^{ \pm} \equiv\left[{ }_{1} R\left(\frac{\partial}{\partial y}, n\right) \widehat{V}(y, \zeta)\right]^{+}-\left[{ }_{2} \widehat{R}\left(\frac{\partial}{\partial y}, n\right) \widehat{V}(y, \zeta)\right]^{-}=\widetilde{F}(y, \zeta),}
\end{gathered}
$$




$$
\left|D_{x}^{\beta} \widehat{V}(x, \zeta)\right| \leq \frac{\text { const }}{1+|x|^{1+|\beta|}}, \quad|\beta|=\overline{0,2},
$$

where ${ }_{j} \chi(x)=\left({ }_{j} \widetilde{X},{ }_{j} \widetilde{X}_{4},{ }_{j} \widetilde{X}_{5}\right)$ is the given vector.

Let $\widehat{V}(x, \zeta)$ be a regular solution of Problem $A(\zeta)$. Taking into account the contact conditions, by virtue of the formulas for general representation of the solution [8] we have

$$
\begin{aligned}
\forall x \in D_{1}: & \left.\widehat{V}(x, \zeta)=\int_{S}{ }_{1} \Phi(x-z, \zeta){ }_{1} \widehat{R} \widehat{V}\right)^{+} d_{z} S- \\
& -\int_{S}\left({ }_{1} \widetilde{\widehat{R}}_{1} \Phi^{*}\right)^{*} \widehat{V}^{+} d_{z} S-\int_{D_{1}}{ }_{1} \Phi_{1} \chi d z \\
\forall x \in D_{2}: & 0=\int_{S}{ }_{1} \Phi\left({ }_{1} \widehat{R} \widehat{V}\right)^{+} d_{z} S-\int_{S}\left({ }_{1} \widetilde{R}_{1} \Phi^{*}\right)^{*} \widehat{V}^{+} d_{z} S- \\
& -\int_{D_{1}}{ }_{1} \Phi_{1} \chi d z, \\
\forall x \in D_{2}: & \widehat{V}(x, \zeta)=-\int_{S}{ }_{2} \Phi\left({ }_{2} \widehat{R} \widehat{V}\right)^{+} d S+\int_{S}\left({ }_{2} \widetilde{\widehat{R}}_{2} \Phi^{*}\right)^{*} \widehat{V}^{+} d S- \\
& -\int_{D_{2}}{ }_{2} \Phi_{2} \chi d z+\int_{S}{ }_{2} \Phi \widetilde{F} d S-\int_{S}\left({ }_{2} \widetilde{\widehat{R}}_{2} \Phi^{*}\right)^{*} \widehat{f} d S, \\
\forall x \in D_{1}: & 0=-\int_{S}{ }_{2} \Phi\left({ }_{1} \widehat{R} \widehat{V}\right)^{+} d S+\int_{S}\left({ }_{2} \widetilde{\widehat{R}}_{2} \Phi^{*}\right)^{*} \widehat{V}^{+} d S- \\
& -\int_{D_{2}}{ }_{2} \Phi_{2} \chi d z+\int_{S} \Phi \widetilde{F} d S-\int_{S}\left({ }_{2} \widetilde{\widehat{R}}_{2} \Phi^{*}\right)^{*} \widehat{f} d S,
\end{aligned}
$$

where the superscripts ${ }^{*}$ and $~$ denote transposition and Lagrange's conjugation, respectively.

It is clear that by substituting $\widehat{V}^{+}$and $\left.{ }_{1} \widehat{R} \widehat{V}\right)^{+}$found from (10) and (12) in (9) and (11) we will solve Problem $A(\zeta)$. It appears that (10) and (12) can be used for constructing approximate values of the unknown vectors.

We introduce the following notations: $z \in S, x \in \mathbb{R}^{3}$,

$$
\begin{aligned}
& { }_{1} \Psi(x, z, \zeta)=\left\|{ }_{\left({ }_{1} \widetilde{R}_{1} \Phi^{*}(x-z, \zeta)\right)^{*}}{ }_{5 \times 5},-{ }_{1} \Phi(x-z, \zeta){ }_{5 \times 5}\right\|_{5 \times 10}, \\
& { }_{2} \Psi(x, z, \zeta)=\left\|\left({ }_{2} \widetilde{R}_{2} \Phi^{*}(x-z, \zeta)\right)^{*}{ }_{5 \times 5},\left.{ }_{-2} \Phi(x-z, \zeta)\right|_{5 \times 5}\right\|_{5 \times 10},
\end{aligned}
$$


$\psi(x, \zeta)=\left\|\psi_{k}\right\|_{10 \times 1}=\left(\widehat{V}^{+},\left({ }_{1} \widehat{R} \widetilde{V}\right)^{+}\right)$is the sought for vector. Now relations (10) and (12) can be rewritten in the form

$$
\begin{aligned}
& \forall x \in D_{2}: \int_{S} \Psi(x, z, \zeta) \psi(z, \zeta) d_{z} S={ }_{1} F(x), \\
& \forall x \in D_{1}: \int_{S}{ }_{2} \Psi(x, z, \zeta) \psi(z, \zeta) d_{z} S={ }_{2} F(x),
\end{aligned}
$$

where

$$
\begin{gathered}
{ }_{1} F(x)=-\int_{D_{1}}{ }_{1} \Phi_{1} \chi d z \\
{ }_{2} F(x)=\int_{D_{2}}{ }_{2} \Phi_{2} \chi d z-\int_{S}{ }_{2} \Phi \widetilde{F} d S+\int_{S}\left({ }_{2} \widetilde{\widehat{R}}_{2} \Phi^{*}\right)^{*} \widehat{f} d S
\end{gathered}
$$

are the given vectors.

Let us construct auxiliary domains and surfaces in the following manner: $\widetilde{D}_{1}$ is a domain bounded by $\widetilde{S}_{1}$ located strictly in $D_{1}$, i.e, $\widetilde{D}_{1} \subset D_{1} ; \widetilde{D}_{2}$ is an infinite domain bounded by $\widetilde{S}_{2}$ located strictly in $D_{2}$. It is clear that $\widetilde{S}_{1} \cap S=\varnothing, \widetilde{S}_{2} \cap S=\varnothing$.

Let $\left\{{ }_{j} x^{k}\right\}_{k=1}^{\infty}, j=1,2$, be a countable, dense everywhere, set of points on the auxiliary surface $\widetilde{S}_{j}, j=1,2$. From (15) and (16) we have

$$
\begin{aligned}
& \int_{S} \Psi\left({ }_{2} x^{k}, z, \zeta\right) \psi(z, \zeta) d_{z} S={ }_{1} F\left({ }_{2} x^{k}\right), \quad k=\overline{1, \infty}, \\
& \int_{S} \Psi\left({ }_{1} x^{k}, z, \zeta\right) \psi(z, \zeta) d_{z} S={ }_{2} F\left({ }_{1} x^{k}\right), \quad k=\overline{1, \infty} .
\end{aligned}
$$

We denote the rows of the matrix ${ }_{j} \Psi$ considered as ten-component vectors by ${ }_{j} \Psi^{1},{ }_{j} \Psi^{2},{ }_{j} \Psi^{3},{ }_{j} \Psi^{4},{ }_{j} \Psi^{5}$ and consider the countably infinite set of vectors

$$
\left\{{ }_{1} \Psi^{l}\left({ }_{2} x^{k}, z, \zeta\right)\right\}_{k=1, l=1}^{\infty, 5} \bigcup\left\{{ }_{2} \Psi^{l}\left({ }_{1} x^{k}, z, \zeta\right)\right\}_{k=1, l=1}^{\infty, 5} .
$$

It is proved that (19) is linearly independent and complete in the space $L_{2}(S)$; i.e., forms the basis in this space.

Let us enumerate set (19) arbitrarily and denote the resulting countable set by

$$
\left\{\psi^{k}(z)\right\}_{k=1}^{\infty} .
$$

We have, for example, performed enumeration like this:

$$
\psi^{k}(z) \equiv{ }_{a_{k}} \Psi^{l_{k}}\left(b_{k} x^{q_{k}}, z, \zeta\right), \quad k=\overline{1, \infty},
$$


where

$$
\begin{gathered}
a_{k}=k-2\left[\frac{k-1}{2}\right], \quad b_{k}=2\left[\frac{k+1}{2}\right]-k+1, \\
l_{k}=\left[\frac{k+1}{2}\right]-5\left[\frac{\left[\frac{k+1}{2}\right]-1}{5}\right], \quad q_{k}=\left[\frac{\left[\frac{k+1}{2}\right]+4}{5}\right] ;
\end{gathered}
$$

$[k]$ is the integer part of the number $k$. It is clear that by virtue of (17) and (18) the scalar product

$$
\left(\psi^{k}, \bar{\psi}\right)=\int_{S} \psi^{k} \psi d S=\left(\psi, \bar{\psi}^{k}\right)
$$

is known for any $k$. Using our notations, we have

$$
\int_{S} \psi^{k} \psi d S={ }_{a_{k}} F_{l_{k}}\left(b_{k} x^{q_{k}}\right), \quad k=\overline{1, \infty} .
$$

Obviously, the complex conjugate system

$$
\left\{\bar{\psi}^{k}(z)\right\}_{k=1}^{\infty}
$$

is also complete.

Now we have to find coefficients $\alpha_{k}, k=\overline{1, N}$ assuming that the meansquare norm

$$
\left\|\psi(z)-\sum_{k=1}^{N} \alpha_{k} \bar{\psi}^{k}(z)\right\|_{L_{2}(S)}
$$

is minimal. As is well-known, for this it is necessary and sufficient that

$$
\left(\psi(z)-\sum_{k=1}^{N} \alpha_{k} \bar{\psi}^{k}(z), \bar{\psi}^{j}(z)\right)=0, \quad j=\overline{1, N} .
$$

Hence we arrive at an algebraic system of equations

$$
\sum_{k=1}^{N} \alpha_{k}\left(\bar{\psi}^{k}, \bar{\psi}^{j}\right)=\left(\psi, \bar{\psi}^{j}\right), \quad j=\overline{1, N},
$$

with the known right-hand side and Gram's determinant differing from zero, which defines coefficients $\alpha_{k}$. Therefore, due to the property of the space $L_{2}(S)$, we have

$$
\lim _{N \rightarrow \infty}\left\|\psi(z)-\sum_{k=1}^{N} \alpha_{k} \bar{\psi}^{k}(z)\right\|_{L_{2}(S)}=0
$$


Let us introduce the notation

$$
\begin{aligned}
& \stackrel{N}{\psi}(z)=\sum_{k=1}^{N} \alpha_{k} \bar{\psi}^{k}(z)
\end{aligned}
$$

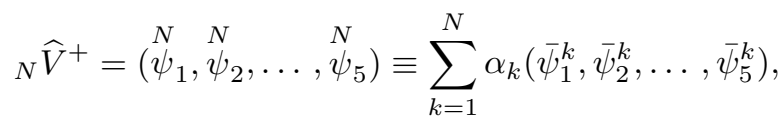

$$
\begin{aligned}
& { }_{N}\left({ }_{1} R_{\tau} \widehat{V}\right)^{+}=\left(\stackrel{N}{\psi_{6}},{ }_{\psi}^{N}, \ldots, \stackrel{N}{\psi_{7}}, \ldots\right) \equiv \sum_{k=1}^{N} \alpha_{k}\left(\bar{\psi}_{6}^{k}, \bar{\psi}_{7}^{k}, \ldots, \bar{\psi}_{10}^{k}\right) .
\end{aligned}
$$

Then we have in the sense of the metric of $L_{2}(S)$ :

$$
\psi(z)=\lim _{N \rightarrow \infty} \stackrel{N}{\psi}(z), \quad \widehat{V}^{+}=\lim _{N \rightarrow \infty} \widehat{V}^{+}, \quad\left({ }_{1} R \widehat{V}\right)^{+}=\lim _{N \rightarrow \infty}{ }_{N}\left({ }_{1} R \widehat{V}\right)^{+} .
$$

Substituting the obtained approximate values in (9) and (11) and denoting the result of the substitution by ${ }_{N} \widehat{V}(x, \zeta)$, we get

$$
\begin{aligned}
\forall x \in D_{1}:{ }_{N} \widehat{V}(x, \zeta)=\int_{S}{ }_{1} \Phi\left(\sum_{k=1}^{N} \alpha_{k}\left(\bar{\psi}_{6}^{k}, \bar{\psi}_{7}^{k}, \ldots, \bar{\psi}_{10}^{k}\right)\right) d S- \\
\quad-\int_{S}\left({ }_{1} \widetilde{\widehat{R}}_{1} \Phi^{*}\right)^{*}\left(\sum_{k=1}^{N} \alpha_{k}\left(\bar{\psi}_{1}^{k}, \bar{\psi}_{2}^{k}, \ldots, \bar{\psi}_{5}^{k}\right)\right) d S-\int_{D_{1}}{ }_{1} \Phi_{1} \chi d z \\
\forall x \in D_{2}:{ }_{N} \widehat{V}(x, \zeta)=-\int_{S}{ }_{2} \Phi\left(\sum_{k=1}^{N} \alpha_{k}\left(\bar{\psi}_{6}^{k}, \bar{\psi}_{7}^{k}, \ldots, \bar{\psi}_{10}^{k}\right)\right) d S+ \\
+\int_{S}\left({ }_{2} \widetilde{R}_{2} \Phi^{*}\right)^{*}\left(\sum_{k=1}^{N} \alpha_{k}\left(\bar{\psi}_{1}^{k}, \bar{\psi}_{2}^{k}, \ldots, \bar{\psi}_{5}^{k}\right)\right) d S- \\
-\int_{D_{2}}{ }_{2} \Phi_{2} \chi d z+\int_{S} \Phi \widetilde{F} d S-\int_{S}\left({ }_{2} \widetilde{R}_{2} \Phi^{*}\right)^{*} \widehat{f} d S .
\end{aligned}
$$

Now for any $\varepsilon \geq 0$ we can give a positive number $N(\varepsilon)$ such that for $N>N(\varepsilon)$ we will have

$$
\left|\widehat{V}(x, \zeta)-{ }_{N} \widehat{V}(x, \zeta)\right|<\varepsilon,
$$

$x \in \bar{D}^{\prime} \subset D ; \widehat{V}(x, \zeta)$ is the exact solution of the problem, i.e.,

$$
\widehat{V}(x, \zeta)=\lim _{N \rightarrow \infty} \widehat{V}(x, \zeta), \quad x \in \bar{D}^{\prime} ;
$$

the convergence to the limit is uniform in $\bar{D}^{\prime}$. 
The method presented here can also be generalized for other more complicated problems.

\section{REFERENCES}

1. V.D. Kupradze, T.G. Gegelia, M.O. Basheleishvili and T.V. Burchuladze, Three-dimensional problems of the mathematical theory of elasticity and thermoelasticity. (Translated from Russian) North-Holland Publishing Company, Amsterdam-New-York-Oxford, 1979; Russian origonal: Nauka, Moscow, 1976.

2. V.D. Kupradze and T.V. Burchuladze, Dynamical problems of the theory of elasticity and thermoelasticity. (Russian) Current Problems in Mathematics, v.7, (Russian) 163-294, Itogi Nauki i Tekhniki Akad. Nauk SSSR, Vsesoyuzn. Inst. Nauchn. i Tekhnich. Inform., Moscow, 1975.

3. T.V. Buchukuri, Some oscillation boundary value problems of elasticity theory with allowance of thermodiffusion. (Russian) Dokl. Akad. Nauk SSSR 235(1977), No. 2, 310-312.

4. "_. ", Boundary value problems of oscillation of moment thermodiffusion. (Russian) Tbiliss. Gos. Univ. Inst. Prikl. Mat. Trudy 7(1980), $5-56$.

5. T.V. Burchuladze, To the theory of dynamic problems of non-classical thermoelasticity. (Russian) Trudy Tbiliss. Mat. Inst. Razmadze, 23(1983), $12-24$.

6. "__, Three-dimensional problems of non-classical thermoelasticity. (Russian) Trudy Tbiliss. Mat. Inst. Razmadze, 25(1984), 4-21.

7. "— " Dynamic problems of the non-classical theory of thermoelasticity. (Russian) Z. Anal. Anwendungen, 3(1984), No. 5, 441-455.

8. T.V. Burchuladze and T.G. Gegelia, The development of the method of potential in the elsticity theory. (Russian) Metsniereba, Tbilisi, 1985.

9. T.V. Burchuladze, To the theory of dynamic problems of thermodiffusion of deformed elastic solid bodies. (Russian) Bull. Acad. Sci. Georgian SSR 89(1978), No.2, 329-332.

(Received 15.01.1993)

Author's address:

A. Razmadze Mathematical Institute

Georgian Academy of Sciences

1, Z. Rukhadze St., Tbilisi, 380093

Republic of Georgia 